\title{
Analysis and Design Framework for Nonlinear Switched Reluctance Machines
}

\author{
A. Stuikys, J. K. Sykulski
}

\begin{abstract}
A new computationally efficient paradigm for the design and analysis of switched reluctance machines is proposed. At the heart of the rapid analysis and design methodology is the reduced order computational method based on a flux tube model which has been refined and extended. It is demonstrated how the improved model enables consistent and accurate analysis and design optimization. Instead of an analytical derivation, an automatic generation of cubic splines is introduced to model the magnetic flux. The flux linkage functions obtained from the improved flux tube method indicate that the method offers good accuracy compared to finite element based analysis, but with significantly improved computational efficiency. The approach is applicable to translating and rotating switched reluctance machines of various topologies and therefore enables rapid design search and optimization of novel topologies.
\end{abstract}

Index Terms -- nonlinear switched reluctance machine, magnetic circuit analysis, flux tube modelling.

\section{NOMENCLATURE}

$A$ : cross-sectional area

$B$ : magnetic field density

$H$ : magnetic field strength

$l$ : flux tube cord length

$R$ : total magnetic circuit reluctance

$r$ : individual flux tube reluctance

$N$ : number of conductor turns

$t$ : tube, flux

$I \quad$ : electric current

$V \quad$ : magnetic field potential at a node

$\alpha$ : flux tube cross-sectional area error

$\psi$ : flux linkage

$\lambda$ : flux tube cord length error

$\pi$ : magnetic field strength error

$\mu$ : magnetic permeability

$\varepsilon \quad$ : average error

$\phi \quad$ : magnetic flux

: estimated value

\section{INTRODUCTION}

N WITCHED reluctance (SR) machines have attracted substantial attention due to their simple and robust construction. SR machines are as versatile, from application point of view, as the well-established induction, DC, and brushless permanent magnet electric machines. The SR technology spans the following topologies: radial flux [1], axial flux [2], transverse flux rotating machines [3], [4],

This work is supported by an EPSRC grant (EP/G03690x/1).

A. Stuikys and J. K. Sykulski are with the Department of Electronics and Computer Science, University of Southampton, Southampton SO17 1BJ, United Kingdom (e-mail: A.Stuikys@soton.ac.uk / jks@ soton.ac.uk). translating (linear) machines [5], [6], and tubular translating (linear) machines [7]. SR machine application areas are as diverse as the more traditional electric machine technologies mentioned earlier. For example, the radial and axial flux machines have been used in many industrial drives, traction motor and pumping applications [8]-[10]. The novel SR linear machines have been used as magnetic propulsion and levitation devices for railway systems in transportation [11]. The tubular linear SR machines were successfully tested in medical applications as artificial heart pump actuators [12].

However, analysis and design of SR machines is a complex task compounded by their non-linear behaviour. Despite some effort [13] the analysis and design calculations have not yet been developed into intuitive analytical tools comparable to the methods available for the more established types of machines such as induction or permanent magnet. The main difficulty with the analysis and design of SR machines is the magnetic nonlinearity caused by the heavily saturated iron parts of the machine circuit. The non-saturating SR machines, as used in some niche applications, are not considered here.

Given the wide variety of topological arrangements of SR machines, which is expected to grow in the future as the demand for the new applications increases [14], it is vital to establish computationally efficient analysis and design methods. The aim is to make the design task more systematic which in turn will open new application areas for the versatile SR electric machine technology.

Reduced order computational methods, the most notable example being the magnetic equivalent circuit (MEC) approach, have been successfully employed in the past to various types of electric machines [15]. The main advantage of the MEC based models is that they are relatively accurate given their computational efficiency. The finite element analysis (FEA) is very useful for accurate analysis of the established electric machine technologies, but it does not offer the cause-and-effect insight when novel and unfamiliar machine topologies are being considered [16]. Therefore, bearing in mind the advantages of the MEC based analysis methods, the design cycle is proposed as illustrated in Fig. 1.

First, a novel topology SR machine is identified and considered for a certain application because it meets some particular requirements imposed by the application, for example: cost, volume and mass, mechanical, etc. Next, the improved flux tube based method, which we propose in this paper, is employed to construct the electromagnetic model of the machine and is subsequently used in conjunction with a design search and optimization algorithm, e.g. the genetic algorithm (GA). Once a set of near optimal solutions (i.e. a Pareto front) is obtained from the previous design cycle step, 
a few designs are identified for further optimization using an order of magnitude more accurate analysis technique, such as FEA. If FEA confirms that indeed these designs are near optimal, the design cycle can conclude as the machine which satisfies all the design constraints has been found. If, however, the FEA proves otherwise, the knowledge and insights gained from the flux tube modelling step are fed back into the novel topology machine generation step and the design cycle is repeated.

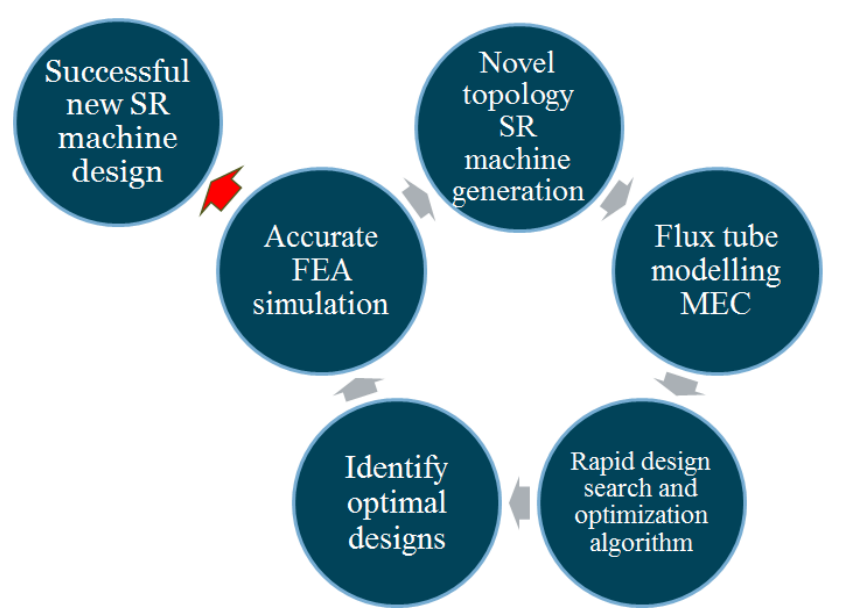

Fig. 1. The proposed SR machine design cycle using a reduced order computational method of flux tubes.

\section{FluX TUBE ModelliNG}

MEC based modelling has been successfully used in the past for rapid analysis of induction and permanent magnet AC machines [17], as well as for SR machines [18]-[20]. In this paper we propose an improved flux tube model for analysis and design of SR machines, which offers computational efficiency and simplicity compared to the traditional analytical derivation of flux tubes as in [20].

First it is necessary to define what is meant by the term flux-tube. Referring to Fig. 2: a flux-tube will have an arbitrary length and an arbitrary cross-sectional area defining a tube in which the magnetic flux is established due to the unequal magnetic potentials $V_{a}$ and $V_{b}$.

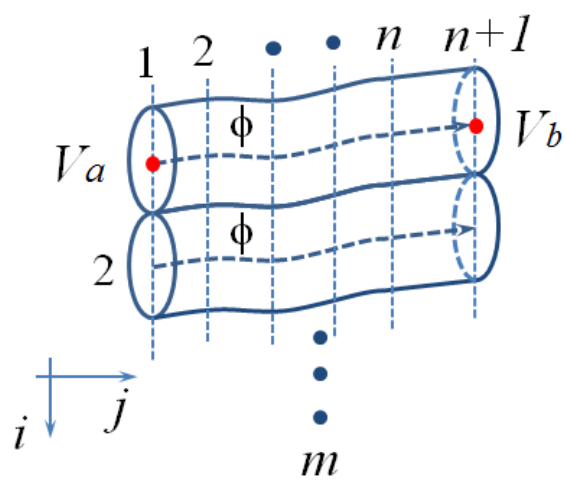

Fig. 2. A system of flux tubes in parallel.

The trajectory of the tube, between the two nodes, can be approximated by a combination of straight line and circular arc segments. The material property, in which the flux-tube exists, is defined by magnetic permeability and will, in general, vary as a function of the magnetic potential along the tube. If the material is air then the permeability of free space is substituted, otherwise the magnetization curve of the material must be used. To form a system of flux tubes in three-dimensions (3D) the tubes are combined to fill the entire 3D space.

Accurate magnetic reluctance estimation of the flux tubes is important as this will determine the flux-linkage functions of the SR machine defined as [16]

$$
\psi=\frac{N^{2} \cdot I}{R} .
$$

In most general terms, and referring to Fig. 2, the flux tube reluctance can be expressed as

$$
R_{a b}=\frac{V_{a}-V_{b}}{\phi}=\int_{0}^{l} \frac{1}{\mu(a, b, l) \cdot A(l)} d l .
$$

The flux-linkage functions is all that is needed to describe the SR machine performance, specifically the speed-torque curve for a rotary machine or thrust force and corresponding linear speed values for a translating machine. From these characteristics the machine output power can readily be found. The procedure will now be illustrated by an example.

\section{A. A Flux Tube Modelling Example}

The analysis of SR machines hinges on the ability to accurately estimate the aligned and unaligned flux-linkage functions as given in (1), which for the saturable machines are always nonlinear for a range of excitation currents [21]. Again, this task is fairly easily accomplished with FEA based methods; however, this approach may be computationally inefficient if a near optimal design of novel and unfamiliar topology machine is sought. Here we propose a method based on the MEC approach. We will show how to obtain the flux linkage functions for an SR machine by constructing approximate flux tube distributions. It is important to note that this approach is equally applicable to the modelling of rotating and translating SR machines.

To illustrate the improved flux-tube based modelling a translating (linear) switched reluctance machine (LSRM) is considered. Figure 3 shows a typical LSRM geometry and the resulting magnetic field distribution obtained from an industry standard FEA. Due to the simple geometric (2D planar) and spatial arrangement of the machine the magnetic flux lines can be approximated using cubic-spline representations of flux-tubes of the true flux paths.

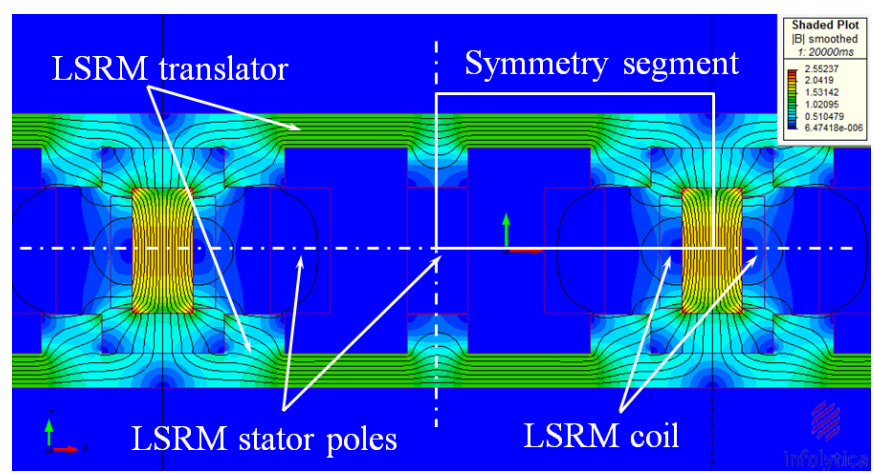

Fig. 3. A double translator linear SR machine topology. 
We start by inspecting the general flux function contour and magnetic field density plot obtained from a standard FEA analysis shown in Fig. 3. Next, due to symmetry, we reduce the analysed magnetic circuit to that of symmetry segment shown in Fig. 3. The proposed flux-tube analysis relies on the ability to approximate the true flux tubes in the magnetic circuit under study using smooth cubic splines.

Zooming into the symmetry segment of the LSRM shown in Fig. 4, we recognize that in most electric machine magnetic circuits of practical importance there will exist the magnetic field equipotential regions.

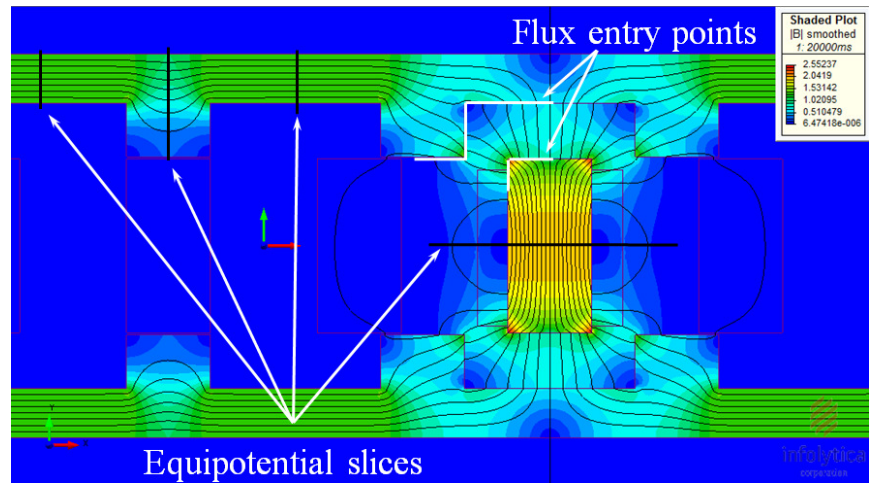

Fig. 4. Subdivision of the symmetry segment into flux tube slices.

Utilizing this knowledge we can construct equipotential slices across the flux paths of the magnetic circuit. Up to this stage we were able to subdivide the circuit into four such slices as shown in Fig. 4. However, an attempt to fit smooth and continuous cubic spline approximations of the real flux tubes between any two neighbouring slices using single set of intermediate points would result in large interpolation errors along the flux paths. This is because the actual flux tubes have geometric discontinuities between the slices. Therefore, it is necessary to subdivide the flux tubes along their lengths even further. It is possible to do so by recognizing the very important property of the flux tubes, that the flux tubes normally enter magnetic material at a right angle to its surface. Thus we are able to construct further slices, this time along the magnetic material surfaces, termed 'flux entry points' as in Fig. 4. Once the flux tube subdivision into slices is complete, the cubic spline interpolation can be performed as illustrated in Fig. 5.

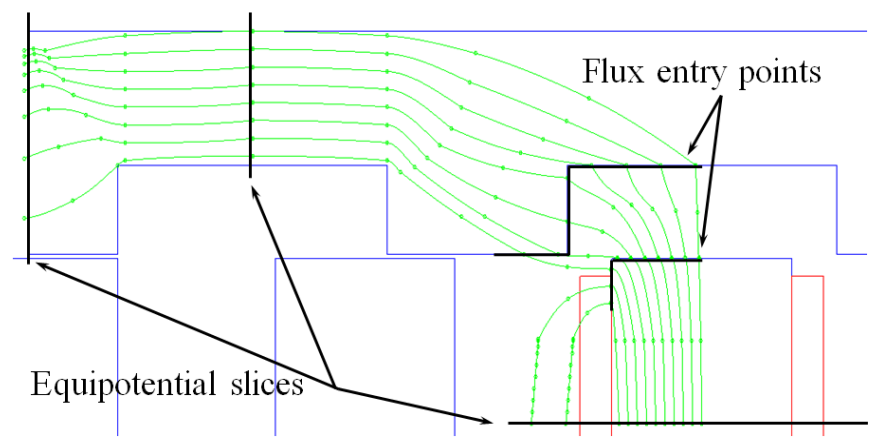

Fig. 5. Cubic-spline approximations of the flux paths for the unaligned translator position.
As is evident from the proceeding discussion and Fig. 4, some prior experience and applying judgment will be helpful when choosing the most representative and convenient coordinate points for the cubic spline interpolation.

Using similar equipotential slice and flux entry point placement techniques the aligned translator position flux-tube approximations are obtained as in Fig. 6. Again, experience and judgement are needed in order to accurately replicate the true flux paths using cubic-spline approximations.

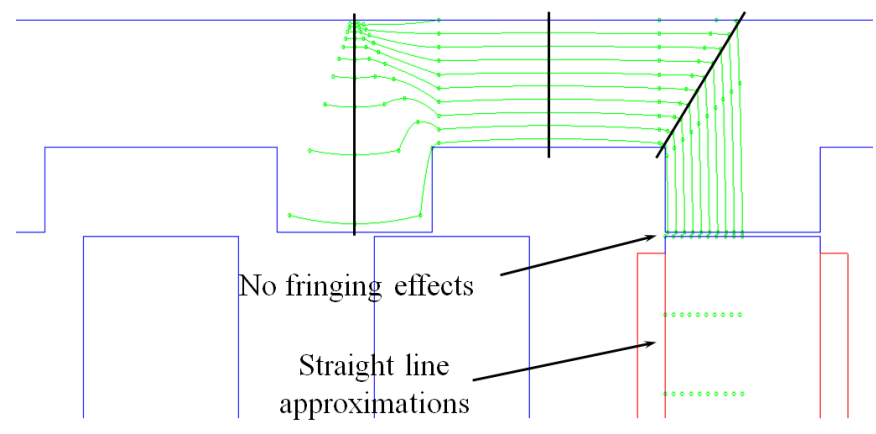

Fig. 6. Aligned translator position flux tubes obtained using cubic-splines.

Some simplifying assumptions are made when fitting the splines in Fig. 6, in particular that there are no fringing or leakage flux effects near the aligned rotor and stator poles as would be expected in a practical machine. Moreover, the energized stator pole flux tubes are approximated using straight lines rather than cubic-splines. Again, this is an approximation, but the resulting errors were found tolerable.

Finally, once the cubic spline approximations to the true flux tube paths are complete, their individual reluctances can be found from (2) as all the geometric information about the tubes is known from the polynomial equation coefficients, specifically the cross-sectional areas, cord lengths and material properties. Therefore, flux linkage functions, as given by (1), for these two translator cases are readily found.

\section{B. Accuracy and Robustness of Flux Tube Modelling}

In order to illustrate the accuracy and robustness of the flux tubes method, from computation and numerical error propagation point of view, analytical formulation is employed. Figure 2 is used to describe what is meant by the system of flux tubes.

1) Air Gap Reluctance: From Fig. 2 it is assumed that the flux tube system is in the air gap or other non-magnetic region of the SR machine. The flux tubes have been subdivided into $n$ slices along their lengths and crosssectional areas of each such slice averaged

$$
A_{j}=\left(A_{j}+A_{j+1}\right) \div 2, \quad j=1,2, \ldots, n
$$

Therefore, the individual reluctance of each of the $m$ flux tubes can be expressed as

$$
R_{a b}=r_{t}=\sum_{j=1}^{n} \frac{l_{j}}{\mu_{0} \cdot A_{j}} .
$$

Now, because the system of flux tubes in Fig. 2 is arranged in parallel, the total reluctance will be 


$$
\frac{1}{R_{\text {parallel }}}=\frac{1}{r_{t 1}}+\frac{1}{r_{t 2}}+\ldots+\frac{1}{r_{t m}}=\sum_{i=1}^{m}\left(\sum_{j=1}^{n} \frac{l_{j}}{\mu_{0} \cdot A_{j}}\right)^{-1} .
$$

As argued in the previous section, the resulting flux tube system of Fig. 5 approximates the FEA solution in Fig. 4 well, but is not exact. The flux tubes method considers discretized systems rather than continuous. Thus it is necessary to take two neighbouring cross-sectional areas of each tube-slice and average them to produce a mean value for that particular flux-tube slice as in (3). This averaging of cross-sectional areas, however, will introduce some numerical error. The error itself will vary from slice to slice, and from tube to tube, and even from system to system. Taking into account the numerical error thus created, (4) can be expressed as follows

$$
\begin{aligned}
r_{t}^{\prime}= & \frac{l_{1} \cdot \varepsilon_{l 1}}{\mu_{0} \cdot A_{1} \cdot \varepsilon_{A 1}}+ \\
& \quad+\frac{l_{2} \cdot \varepsilon_{l 2}}{\mu_{0} \cdot A_{2} \cdot \varepsilon_{A 2}}+\ldots+\frac{l_{n} \cdot \varepsilon_{l \cdot n}}{\mu_{0} \cdot A_{n} \cdot \varepsilon_{A n}} .
\end{aligned}
$$

It is assumed that each error term $\varepsilon$ in (6) will be in the region of, and unlikely to exceed, \pm 1.1 for the averaged crosssectional areas and cord lengths of each slice. If the number of terms $n$ in (6) is increased, the errors resulting from the estimated cord lengths and cross-sectional areas of the tube slices can be averaged, namely

$$
r_{t}^{\prime}=\left(\frac{l_{1}}{\mu_{0} \cdot A_{1}}+\frac{l_{2}}{\mu_{0} \cdot A_{2}}+\ldots+\frac{l_{n}}{\mu_{0} \cdot A_{n}}\right) \times \frac{\varepsilon_{\text {laav }}}{\varepsilon_{\text {A.av }}} .
$$

For convenience, the averaged errors of the cross sectional areas and cord lengths of each slice in (7) can be written as

$$
A_{j} \cdot \varepsilon_{\text {A.av }}=A_{j}+A_{j} \cdot \alpha ; \quad l_{j} \cdot \varepsilon_{l \cdot a v}=l_{j}+l_{j} \cdot \lambda .
$$

It is now possible to show what impact the errors of the estimated geometries will have on the reluctance value of the whole system of tubes of Fig. 2. Rewriting (6) in terms of (8)

$$
\begin{aligned}
r_{t}^{\prime} & =\frac{l_{1} \cdot(1+\lambda)}{\mu_{0} \cdot A_{1} \cdot(1+\alpha)}+ \\
+ & \frac{l_{2} \cdot(1+\lambda)}{\mu_{0} \cdot A_{2} \cdot(1+\alpha)}+\ldots+\frac{l_{n} \cdot(1+\lambda)}{\mu_{0} \cdot A_{n} \cdot(1+\alpha)}= \\
& =\left(\sum_{j=1}^{n} \frac{l_{j}}{\mu_{0} \cdot A_{j}}\right) \times \frac{(1+\lambda)}{(1+\alpha)}
\end{aligned}
$$

and rewriting (5) in terms of (9)

$$
\frac{1}{R_{\text {parallel }}^{\prime}}=\sum_{i=1}^{m}\left(\sum_{j=1}^{n} \frac{l_{j}}{\mu_{0} \cdot A_{j}}\right)^{-1} \times \frac{(1+\alpha)}{(1+\lambda)}
$$

results in the total reluctance of the system of tube-slices in series and combined in parallel to be

$$
R_{\text {parallel }}^{\prime}=\left(\sum_{i=1}^{m}\left(\sum_{j=1}^{n} \frac{l_{j}}{\mu_{0} \cdot A_{j}}\right)^{-1}\right)^{-1} \times \frac{(1+\lambda)}{(1+\alpha)} .
$$

The result in (11) indicates that the estimated parallel reluctance of the air gap tubes will scale linearly with the quotient of the two errors. Thus it could be argued that if the two error terms are both positive or both negative this will tend to minimize the total error of the parallel reluctance. The worst case scenario occurs if the two errors are of equal magnitude but opposite sign, that is the cross-sectional areas are underestimated whilst the cord lengths of the tubes are overestimated, or vice versa. Even the worst case scenario is considered to be tolerable provided the errors $\alpha$ and $\lambda$ are not larger than $\pm 10 \%$ as stated earlier. Under such conditions the total error can be no more than $\pm 22 \%$.

2) Iron Circuit Reluctance: In a similar way the reluctance of the magnetically nonlinear iron circuit of the SR machine can be estimated and effects of errors accounted for. Consider Fig. 2 again, this time however with the magnetic permeability no longer constant but varying according to the magnetization curve of the material. Therefore (4) may be rewritten, taking saturation into account, as

$$
R_{a b}=\frac{H_{a b} \cdot l_{a b}}{B_{a b} \cdot A_{a b}}=r_{t}=\sum_{j=1}^{n} \frac{H_{j} \cdot l_{j}}{B_{j} \cdot A_{j}} .
$$

Assuming that the flux of each tube is of constant value the magnetic field density occurring at each slice is

$$
B_{j}^{\prime}=\frac{\phi}{A^{\prime}{ }_{j}}=\frac{\phi}{A_{j}(1+\alpha)}
$$

and from (13) the following can be deduced

$$
\begin{aligned}
& \text { if } \quad \alpha>0 \text { then } \quad B^{\prime}{ }_{j}<B_{j} \\
& \text { if } \quad \alpha<0 \text { then } \quad B^{\prime}{ }_{j}>B_{j} .
\end{aligned}
$$

In other words, if the error is positive the magnetic field density will be underestimated compared to the true value and vice versa. From the magnetization curve of Fig. 7 it follows that the relationship between the erroneous magnetic field density estimate and the resulting magnetic field strength can be stated as

$$
\begin{aligned}
& \text { if } \quad B^{\prime}{ }_{j}<B_{j} \text { then } H^{\prime}{ }_{j}<H_{j} \\
& \text { if } B^{\prime}{ }_{j}>B_{j} \text { then } H^{\prime}{ }_{j}>H_{j} \text {. }
\end{aligned}
$$

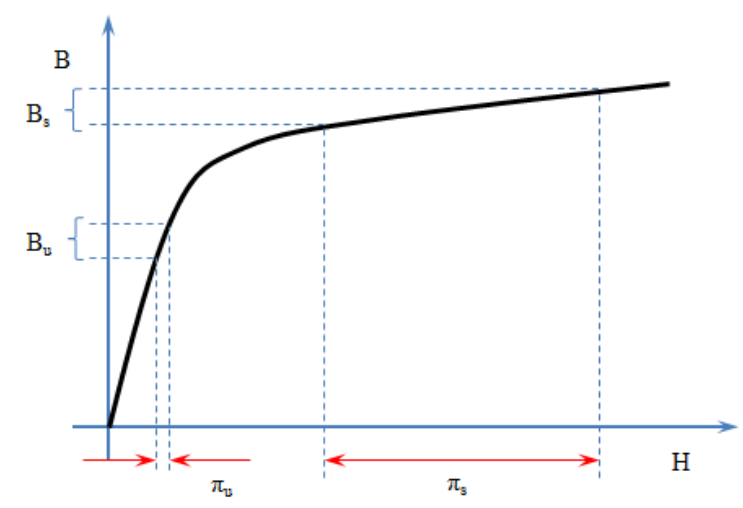

Fig. 7. A typical magnetization curve of a saturable iron circuit and the resulting worst case errors in the non-saturated and saturated regions.

The resulting erroneous magnetic field strength for each of the flux-tube slices along the lines of (8) can be expressed, for convenience, as

$$
H^{\prime}{ }_{j}=H_{j}+\pi \cdot H_{j}=H_{j}(1+\pi)
$$


The estimated values in the denominators of (12) imply that

$$
B^{\prime}{ }_{j} \cdot A^{\prime}{ }_{j}=\frac{\phi}{A^{\prime}{ }_{j}} \times A^{\prime}{ }_{j}=\frac{\phi \times A_{j}(1+\alpha)}{A_{j}(1+\alpha)}=\phi
$$

which is trivial, however it does show that the estimated flux, that is the denominator in (12), is not affected by the crosssectional area error $\alpha$.

Equation (12) can now be expressed in terms of (16), also remembering to include the tube slice cord length error term derived earlier, as follows

$$
\begin{gathered}
r_{t}^{\prime}=\frac{H_{1} \cdot(1+\pi) \times l_{1} \cdot(1+\lambda)}{B_{1} \cdot A_{1}}+ \\
+\frac{H_{2} \cdot(1+\pi) \times l_{2} \cdot(1+\lambda)}{B_{2} \cdot A_{2}}+\ldots+ \\
\quad+\frac{H_{n} \cdot(1+\pi) \times l_{n} \cdot(1+\lambda)}{B_{n} \cdot A_{n}}= \\
=\left(\sum_{j=1}^{n} \frac{H_{j} \cdot l}{B_{j} \cdot A_{j}}\right) \times(1+\pi) \cdot(1+\lambda)
\end{gathered}
$$

and the parallel reluctance of the system of tubes in the iron circuit is

$$
\begin{aligned}
& R_{\text {parallel }}^{\prime}= \\
& =\left(\sum_{i=1}^{m}\left(\sum_{j=1}^{n} \frac{H_{j} \cdot l_{j}}{B_{j} \cdot A_{j}}\right)^{-1}\right)^{-1} \cdot(1+\pi) \cdot(1+\lambda) .
\end{aligned}
$$

Equation (19) indicates that the cross-sectional area estimation error $\alpha$ has no influence on the final result of the total reluctance of the iron circuit. However, a new error term appears in (19) due to the estimation of magnetic field strength from the non-linear iron magnetization curve. Therefore, the total saturating iron reluctance will scale as a product of the two errors. In contrast to the total air gap reluctance, as in (11), the iron circuit reluctance value is affected less by the equal magnitude but opposite signs of the errors (that is when one is positive and the other is negative). The worst case scenarios occur when both error terms are of equal magnitude and either both positive, or both negative.

Attention is now turned towards the discussion of the errors in (19) when the iron circuit is in the non-saturated and when it is in the fully saturated regions of the magnetization curve since steady-state SR machine operation takes place in both of these regions. As can be seen from Fig. 7, the error term for the magnetic field strength will be very small when the flux-linkage values of the SR machine are estimated at low phase excitation currents. The situation is very different when flux-linkage values of the SR machine are estimated at high phase excitation current levels where most of the iron circuit is fully saturated. At this condition the $\pi$ error term is significantly larger, even if uncertainty associated with the magnetic field density value is the same as in the nonsaturated region.

Due to the magnetic circuit design of the SR machine used here for illustrative purposes, the air gap and iron circuit components are arranged magnetically to be in series. Thus for the both the aligned and unaligned flux-linkage function estimations, the reluctances given by (11) and (19) will be additive, while the error terms for the unaligned flux-linkage curve will be order of magnitude smaller compared to the error terms of the aligned flux-linkage curve. This effect is due to the point made above, but to repeat: the saturated reluctance error of the aligned SR machine circuit will be exacerbated due to error terms in (19), whereas in (11) the error will be negligible as the air region's length is small as in Fig. 6. On the other hand, the reluctance error of the unaligned SR machine circuit will be made worse due to increased error terms in (11) as the air-gap region's length is large, whereas in (19) the error will be relatively much smaller because of the non-saturated state of the iron as in Fig. 5.

The preceding error analysis of the flux tube approach, and in particular expressions (11) and (19), can be directly compared to the 'tubes-and-slices' (TAS) method used to analyse electric and magnetic fields in linear media [22]. The analytical TAS derivation, in addition to using the tubes, makes use of the construction of a system of slices along the lines of Fig. 2, leading to the creation of dual bounds. The tubes result in a lower bound of the permeance, whereas the slices in an upper bound. There is similarity to the calculation of a resistance or capacitance, with the analogue of the permeability, $\mu$, being the conductivity, $\sigma$, or permittivity, $\varepsilon$, respectively. The bounds are guaranteed and thus the true answer always lies between the two values. Taking an average often results in a good numerical approximation.

It may be possible to adapt the flux tube approach if the resulting error of the system of tubes is to be minimized. A possible strategy is to compare the flux-linkage function based on a particular flux-tube system with a FEA solution and use the numerical error found to correct the subsequently generated systems of flux tubes. Such a strategy would be likely to be most effective when a large number of flux tube systems is being generated, as in GA based optimization.

\section{FluX TUbe MOdEl RESUlts}

The proposed flux tube model has been applied to a wide range of SR machine geometries to test if the new method consistently and accurately predicts the performance. To accomplish this, six machine design parameters were selected and varied in order to generate distinct machine geometries. Referring to Fig. 3, the following variables were designated: translator back iron thickness, translator pole height, stator and translator pole pitch, the pole width, number of turns per coil, machine stack thickness.

Figure 8 compares the aligned and unaligned flux-linkage functions, assuming flat-topped current profile as in [16], obtained with the improved flux tube method and using commercial FEA. The unaligned flux-linkage functions from both methods compare very well and even such effects as the onset of saturation at higher phase currents, 150A and slightly above, are also captured by the flux tube model. Clearly the unaligned flux-linkage function is linear up to the peak flat-topped current, $150 \mathrm{~A}$ in this case. Therefore the saturation effects and, more importantly, the numerical 
discrepancy from the FEA result will not affect the machine performance prediction in any noticeable way.

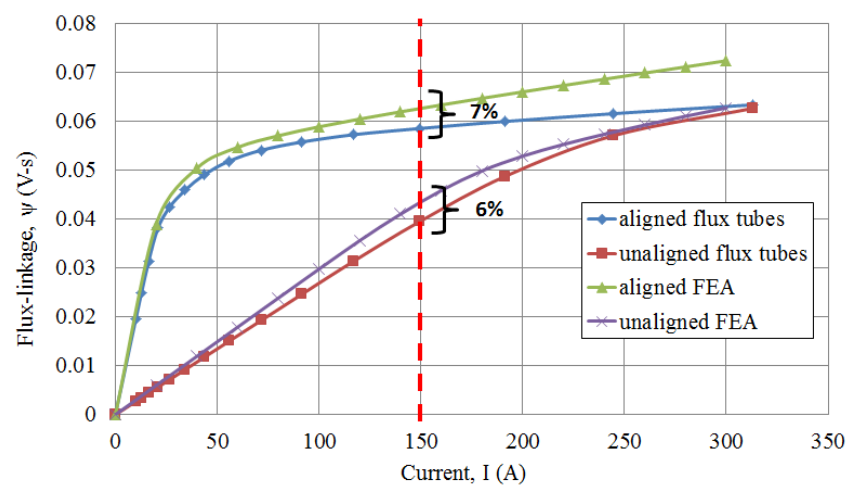

Fig. 8. Flux-linkage functions using the flux-tube method and FEA.

The aligned flux-linkage functions obtained from the two methods show some numerical discrepancies, although they are not large. Assuming that the aligned flux-linkage function obtained using FEA software is correct, the flux tube method underestimates the value by $7 \%$ at the peak current of $150 \mathrm{~A}$. The error is reassuringly low, bearing in mind that the flux tube model of Fig. 6 used only 12 flux-tube slices, which is a coarse subdivision compared to the fine mesh used in FEA. Even more importantly, the aligned flux-linkage function obtained by the flux tube method preserves the 'true' shape of the aligned flux-linkage function as obtained with FEA. This fact, already observed and reported in literature [21] and evident from Fig. 9, has far reaching implications and greater importance - when determining instantaneous torque and phase current waveforms - than the exact numerical answer at only saturated or only unsaturated machine states. Finally as expected - the accuracy of the approximate prediction becomes worse in the heavily saturated region of the aligned position, but this is of no practical consequence as the operating current is above the peak value; this part of the curve has been included for completeness, but should be disregarded in the context of the design process.

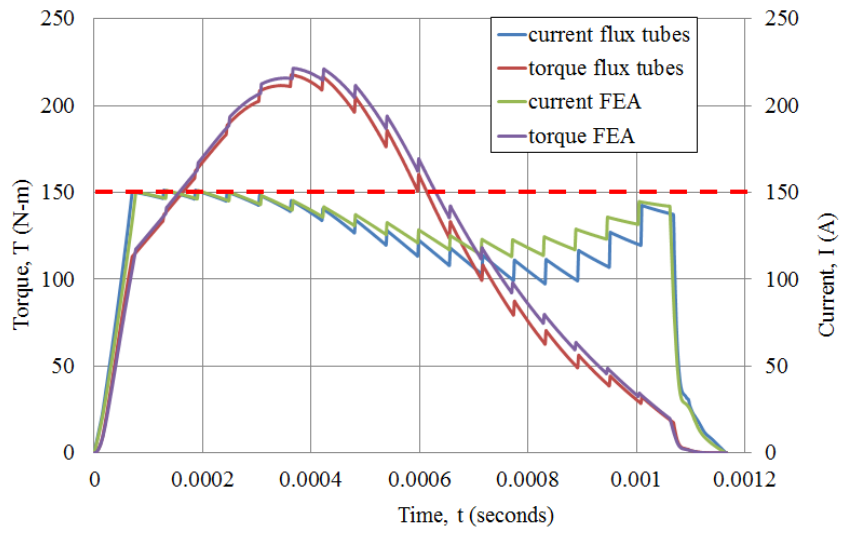

Fig. 9. Comparison of current and instantaneous torque waveforms obtained with the flux tube method and FEA.

Consequently, from a visual comparison of the functions in Fig. 8, it is evident that the numerical discrepancies are not as significant compared to the similarity of shapes. Drawing the knowledge gained from the above presented analytical derivation of the flux tube method, it is now clear that the answer given by (19) is more sensitive to the estimated magnetic field strength values in the saturated region than to the non-saturated region of Fig. 7. In this particular case the total circuit reluctance is overestimated, which is conservative, giving the lower aligned flux-linkage function as in Fig. 8.

The instantaneous torque and phase current waveform comparison is important when assessing the improved flux tube method accuracy. Similarly, it is very useful to compare the speed-torque characteristics of the translating SR machine. The machine geometry is first converted from the translating machine domain to the rotating machine domain, as described in [16], to facilitate ease of comparison. Fig. 10 compares speed-torque characteristic envelopes obtained by the flux-tube method and FEA.

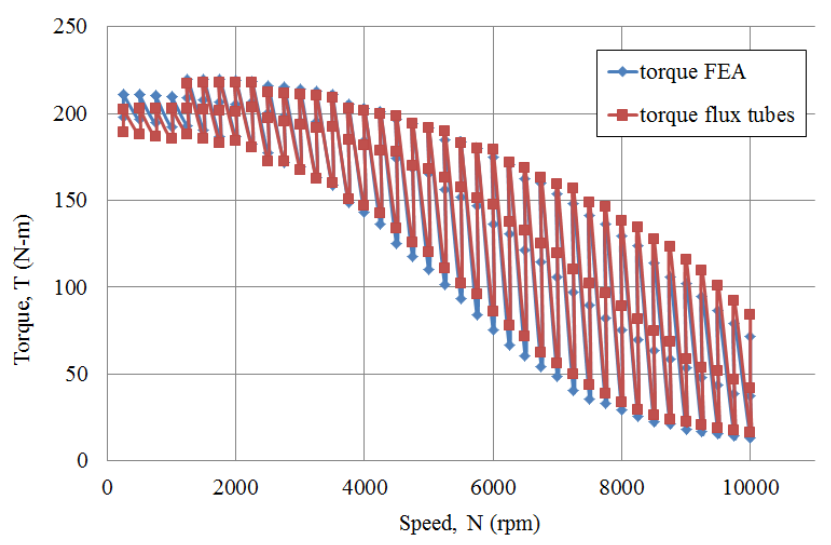

Fig. 10. Speed-torque envelopes of the flux tube method and FEA.

Again, from the visual comparison of superimposed envelopes, it is evident that the flux tube method accurately and consistently predicts the average torque values, consistent with the FEA results, over a wide speed range. In a similar way the speed-power envelopes of the SR machine are compared in Fig. 11.

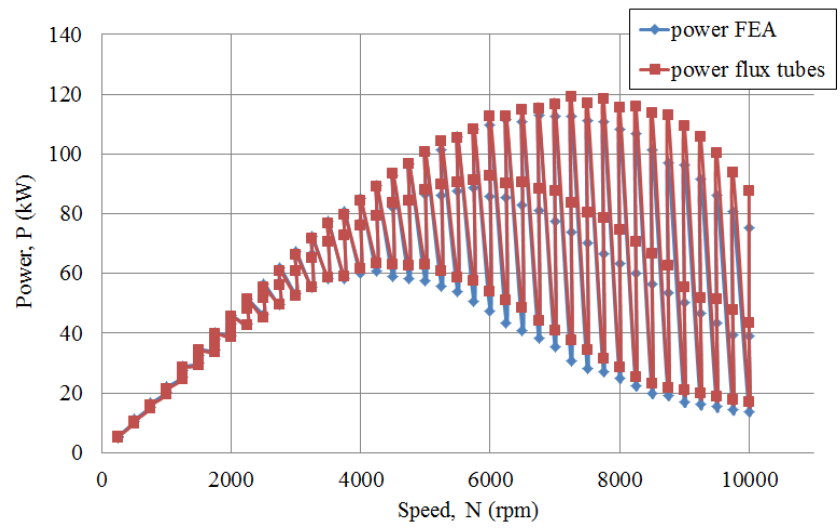

Fig. 11. Speed-power envelopes of the flux tube method and FEA.

As argued in this paper, the method of the flux tubes has been demonstrated to possess the necessary consistency and accuracy, while being general in scope of applications. It can 
be applied to rotating SR machines and to a wide range of SR machine topologies more generally.

A possible enhancement of the method might include a direct incorporation of the dual bounds concepts by adding a second calculation based on slices, as in the original tubesand-slices approach. However, this needs to be considered with care as the additional effort and associated extended computing times may not necessarily be justified since the application of flux tubes alone appears to provide sufficient accuracy while preserving computational efficiency necessary for the rapid design purposes of Fig. 1.

Finally, it should be noted that the purpose of this work is to improve the practicality of the design process, normally relying on finite element modelling, by supplementing it with a much more computationally efficient methodology exploiting semi-analytical flux-tube field description. The appropriateness of the finite element approach has been proven before, including experimental verification. In this work we focus on improving the speed of numerical analysis while preserving the original level of accuracy.

\section{CONCLUSIONS}

The paper has introduced a new analysis and design paradigm in application to SR machines. It is believed that the proposed design cycle will enable systematic and computationally efficient effort to be expended when novel and unfamiliar topology SR machines are considered for new applications. To speed up the design search and optimization task an improved flux tube modelling has been proposed. The improved method enables rapid analysis and design of electromechanical devices, in particular efficient estimation of flux-linkage functions used to describe the operation of SR machines. It has been demonstrated that the flux tube method can relatively accurately and consistently predict these flux linkage functions, including such effects as magnetic saturation and flux leakage. It is concluded that the inaccuracies generated by the flux tube method are small and do not introduce unacceptable errors in the values of predicted currents and torques, both instantaneous and average. Therefore the method is suitable for rapid initial design search and optimization of SR machines of various topologies.

\section{REFERENCES}

[1] M. Takeno, A Chiba, N. Hoshi, S. Ogasawara, M. Takemoto, and M. Azizur Rahman, "Test results and torque improvement of the $50-\mathrm{kW}$ switched reluctance motor designed for hybrid electric vehicle," IEEE Trans. Industry Applications, vol. 48, pp. 1327-1334, Aug. 2012.

[2] A. Labak, and N. C. Kar, "Novel approaches towards leakage flux reduction in axial flux switched reluctance machines," IEEE Trans. on Magnetics, vol. 49, no. 8, August 2013.

[3] Y. Li, H. Li, and Z. Liu, "Design and implement of novel transverse flux switched magnetic energy motor combined with control system," Proceedings of IEEE International Conference on Mechatronics and Automation, August 4-7, Takamatsu, Japan 2013.

[4] I. A. Viorel, M. Crivii, L. Lowenstein, L. Szabo, and M. Gutman, "Direct drive systems with transverse flux reluctance motors," ACTA Electrotechnica, vol. 45, pp. 33-40, no. 3, 2004.

[5] L. Byeong-Seok, "Linear switched reluctance machine drives with electromagnetic levitation and guidance systems," Doctoral Thesis, Virginia Polytechnic Institute and State University, November, 2000.
[6] U. S. Deshpande, J. J. Cathey and E. Richter, "High-force density linear switched reluctance machine," IEEE Transactions On Industry Applications, vol. 31, pp. 345-352, no. 2, March/April 1995.

[7] I. Mahmoud, and H. Rehaoulia, "Design, nonlinear modelling and performances of a biomedical system," Int. J. of Applied Electromagnetics and Mechanics, vol. 50, no. 1, pp. 127-143, 2016.

[8] W. F. Ray, P. J. Lawrenson, R. M. Davis, J. M. Stephenson, N. N. Fulton, and R. J. Blake, "High-performance switched reluctance brushless drives", IEEE Trans. On Industry Applications, vol. IA-22, pp. 722-730, no. 4, Aug. 1986.

[9] RapidSR, Ricardo [Online]. Available at: www.ricardo.com.

[10] SRDrives Ltd. [Online]. Available at: www.srdrives.com.

[11] L. Kolomeitsev, D. Kraynov, S. Pakhomin, F. Rednov, E. Kallenbach, V. Kireev, T. Schneider, and J. Bocker, "Linear switched reluctance motor as a high efficiency propulsion system for railway vehicles," Int. Symp. on Power Electronics, Electrical Drives, Automation and Motion, SPEEDAM 2008.

[12] J. Llibre, N. Martinez, P. Leprince and B. Nogarede, "Analysis and modelling of linear-switched reluctance for medical application", Actuators, vol. 2, pp. 27-44, 2013.

[13] R. Krishnan, R. Arumugam and J. F. Lindsay, "Design procedure for switched reluctance motors," IEEE Trans. on Industry Applications, vol. 24, no. 3, pp. 456-461, June 1988.

[14] E. El-Kharashi, "Improving the energy conversion process in the switched reluctance motors," Int. J. of Applied Electromagnetics and Mechanics, vol. 41, no. 4, pp. 375-387, 2013.

[15] V. Ostovic, Dynamics of Saturated Electric Machines. ISBN-13: 9781-4613-8935-4, New York, Springer-Verlag, 1989.

[16] R. Krishnan, Switched Reluctance Motor Drives: Modelling, Simulation, Analysis, Design, and Applications. ISBN: 0849308380, CRC Press LLC, Boca Raton Florida, 2001.

[17] M. Amrhein and P. T. Krein, "3-D magnetic equivalent circuit framework for modeling electromechanical devices," IEEE Trans. on Energy Conversion, vol. 24, pp. 397-405, no. 2, June 2009.

[18] H.C. Roters, Electromagnetic Devices, John Wiley \& Sons Inc., 1941.

[19] J. Corda and J.M. Stephenson, "Analytical estimation of the minimum and maximum inductances of a double-salient motor," Proc. of Int. Conf. on Stepping Motors and Systems, Leeds, pp. 50-59, Sept. 1979.

[20] J. F. Tabriz and J. W. Finch, "Two-Dimensional leakage flux estimation in switched reluctance motors," Fourth Int. Conf. on Electrical Machines and Drives, 1989.

[21] T. J. E. Miller, "Nonlinear theory of the switched reluctance motor for rapid computer-aided design," IEE Proc., vol. 137, no. 6, Nov. 1990.

[22] J. K. Sykulski, "Dual field modelling using tubes and slices", Advances in Engineering Software, vol. 21, pp. 21-26, Elsevier Science Limited, 1995.

\section{BIOGRAPHIES}

Aleksas Stuikys received the B.Sc. degree in Mechanical Engineering from Oxford Brookes University, Oxford, UK, in year 2009. He received the M.Sc. degree in Advanced Engineering Design in year 2011 from the same institution. After a number of years working in automotive industry as an engineer he returned to academia to pursue the doctoral degree at the Institute for Complex Systems Simulation, School of Electronics and Computer Science, University of Southampton, Southampton, UK. His research interests span the fields of propulsion systems, including electric traction motors; their modelling, simulation and design for the electric and hybrid vehicles and for the sustainable transport in general. This research also includes the modelling, design and control aspects of switched reluctance machines and traction systems.

Jan Sykulski is Professor of Applied Electromagnetics at the University of Southampton, UK. His personal research is in development of fundamental methods of computational electromagnetics, power applications of high temperature superconductivity, simulation of coupled field systems and design and optimisation of electromechanical devices. He has published over 370 scientific papers and co-authored four books. He is founding Secretary of International Compumag Society, Visiting Professor at universities in Canada, France, Italy, Poland and China, Editor of IEEE Transactions on Magnetics, Editor-in-chief of COMPEL (Emerald) and member of International Steering Committees of several international conferences. He is Fellow of IEEE (USA), Fellow of the Institution of Engineering and Technology (IET), Fellow of the Institute of Physics (IoP), Fellow of the British Computer Society (BCS), Doctor Honoris Causa of Universite d'Artois, France, and has an honorary title of Professor awarded by the President of Poland. http://www.ecs.soton.ac.uk/info/people/jks . 